\title{
Two-mode dynamo as a oscillator with memory
}

\author{
Gleb Vodinchar ${ }^{1, *}$ \\ ${ }^{1}$ Institute of Cosmophysical Research and Radio Wave Propagation FEB RAS \\ 7 Mirnaya Str., Paratunka, Kamchata, 684043 Russia
}

\begin{abstract}
The hereditary two-mode model of the $\alpha \omega$-dynamo proposed earlier by the author is studied from the point of view of the theory of oscillators. It is shown that this approach allows one to perform a qualitative analysis of the model's behavior and predict possible dynamic regimes without numerical simulation. Examples of comparison of the results of qualitative analysis and modeling are given.
\end{abstract}

\section{Introduction}

The real cosmic dynamo-systems is a oscillating systems. For dynamo of stellars and planets an reversal phenomenon is observed, and the series of reversals contain both regular and chaotic components $[1,2]$.

The extremely simplified dynamo models are interesting in which the spatial structure of the fields is ignoried, but reversals regimes (regular or/and chaotic) arise. We have in mind are completely deterministic models, without parameter fluctuations or noises. Such models are dynamical systems of small dimension and describe only the basic phenomenology of dynamo. The such systems primarily include the Rikitake system [3] in which chaotic reversal arise, but this system is laboratory, its derivation from the MHD equations is very problematic. Another example of a classical system with stationary regimes and regimes of regular and chaotic reversals is the classical Lorentz system [4]. The Lorenz system as a dynamo model was proposed by [5] for explanation of the chaotic component of the solar cycle.

Earlier, we considered some integro-differential generalization of the Lorenz system as a model of a two-mode dynamo with hereditary quenching of $\alpha$-effect [6]. In this paper, we will study it as an oscillator.

\section{Mathematical model}

The simplest two-mode model of temporal variations of an axisymmetric magnetic field can be written in the following dimensionless form [6]:

$$
\begin{aligned}
& \frac{d x}{d t}=\left(\sigma-\frac{\xi}{s^{2}} z\right) y-\sigma x, \\
& \frac{d y}{d t}=(D-z) x-y, \\
& z(t)=\int_{0}^{t} K(t-\tau) q(x(\tau), y(\tau)) d \tau,
\end{aligned}
$$

*e-mail: gvodinchar@ikir.ru 
where $x(t)$ and $y(t)$ - is the scalar amplitudes of toroidal and poloidal modes, $\sigma-$ is the ratio of diffusion times of toroidal and poloidal modes, $\xi$-is the ratio of efficiencies of the $\alpha$-effect for the poloidal and toroidal fields, $s-$ is the ratio of typical values of toroidal and poloidal fields, $D-$ is the dynamo-number.

The timescale in (1) - is the diffusion time of poloidal field. It is known, that if we take the one toroidal and one poloidal modes of free decay of a magnetic field with the same spatial scales, then the poloidal mode has an eigenvalue smaller. Therefore, in the future, we believe that $\sigma>1$. It is clear that the stationary point $(0,0,0)$ will be unstable if and only if $D>1$. It is in this case that the dynamo will be «working». Therefore, in the future, we assume that $D>1$.

In the system (1) $q(x, y)$ - is a quadratic function, and expression for $z(t)$ is specify the model of feedback - hereditary quenching of $\alpha$-effect by field. $K(\cdot) \geq 0$ - some kernel, with the property $K(+\infty)=0$.

The system (1) is closed by the initial conditions $x(0)=x_{0}, y(0)=y_{0}$. The question of the existence and uniqueness of a solution to the system (1) for given initial conditions is not considered in this paper.

In what follows, we will always assume that the toroidal field is zero at the initial time, i.e. $x_{0}=0$. This follows from the very idea of dynamo $\alpha \omega$-dynamo [7]. During the initial moment, the existence of a poloidal field of dipole type is supposed. During the differential rotation, the magnetic field lines of a highly conducting medium curl around the axis of rotation ( $\omega$-effect); this leads to the appearance of the toroidal field in the convective zone of a star or a planet liquid core. To close the cycle, it is necessary to get a new poloidal field from this toroidal one. It is assumed that this is due to the breaking of mirror symmetry flows in the convection zone. Turbulent mirror-asymmetric flow generates effective the electromotive force in the direction of the toroidal field ( $\alpha$-effect).

The integro-differential system (1) is a simplest model of the $\alpha^{2} \omega$-dynamo. In particular, the system describes: $\alpha^{2} \omega$-dynamo, if $\xi \neq 0$ and $s^{2} \sigma>\xi D ; \alpha^{2}$-dynamo, if $\xi \neq 0$ and $s^{2} \sigma=\xi D ; \alpha \omega$-dynamo, if $\xi=0$ [6]. In this paper, we restrict ourselves to the $\alpha \omega$-dynamo case only, i.e. $\xi=0$. Then the model will take the form:

$$
\begin{aligned}
& \frac{d x}{d t}=\sigma y-\sigma x, \\
& \frac{d y}{d t}=(D-z) x-y, \\
& z(t)=\int_{0}^{t} K(t-\tau) q(x(\tau), y(\tau)) d \tau,
\end{aligned}
$$

Now consider the possible form of the quadratic function $q(x, y)$. The following two expressions have a physical meaning:

1. $q(x, y)=x y-$ is the helicity of the magnetic field;

2. $q(x, y)=s x^{2}+\frac{1}{s} y^{2}-$ is the energy of the magnetic field.

In this paper we consider the case of quenching of the $\alpha$-effect energy, i.e.

$$
z(t)=\int_{0}^{t} K(t-\tau) x(\tau) y(\tau) d \tau
$$

Now we transform the system (2) to the form of an oscillator. 


\section{Oscillator}

We differentiate the first equation of system (2) in time, use the second equation of this system and equation (3). After some transformations, we get the following system:

$$
\begin{aligned}
& \frac{d^{2} x}{d t^{2}}+(1+\sigma) \frac{d x}{d t}+\frac{K(0)}{2} x^{3}-[\sigma(D-1)-w(t)] x=0, \\
& w(t)=\int_{0}^{t} J(t-\tau) x^{2}(\tau) d \tau,
\end{aligned}
$$

where a new kernel

$$
J(\cdot)=\frac{K^{\prime}(\cdot)}{2}+\sigma K(\cdot)
$$

The initial conditions for systems (2) and (5) are related by equalities:

$$
x(0)=0, \quad y(0)=y_{0}, \quad x^{\prime}(0)=\sigma y_{0} .
$$

The system (5) can be considered as a dissipative oscillator with hereditary potential

$$
\begin{aligned}
& U(x)=A x^{4}-B x^{2}, \\
& A=K(0) / 8 \geq 0, \quad B=\frac{\sigma(D-1)}{2}-\frac{1}{2} \int_{0}^{t} J(t-\tau) x^{2}(\tau) d \tau .
\end{aligned}
$$

Consider first the case $K(0) \neq 0$, i.e. $A>0$. This means that feedback occurs instantly without delay. The shape of the potential $U(x)$ is determined by the sign of the coefficient $B$ (Fig. 1).

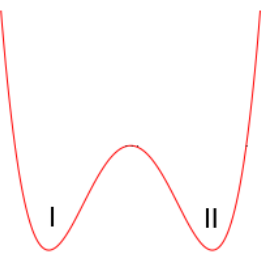

$\mathrm{B}>0$

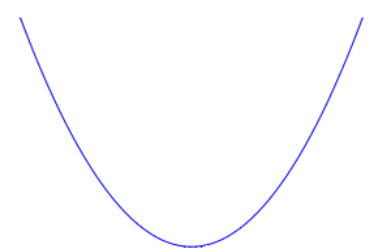

$B<0$

Figure 1. The shape of potential $U(x)$ for oscillator (5) in case $K(0) \neq 0$.

Considering the system (2) as the oscillator (5), we can describe various possible dynamic regimes without specifying the kernel $K(\cdot)$ and without numerical simulation.

Suppose that for some basic kernel $K(t)$, the new kernel $J(t)<0$ if $t \geq 0$. Then all the time $B>0$. Therefore, losing energy, the oscillator will fall into one of the potential wells and there will be a regime of stable field generation without oscillations.

If $J(t)$ is alternating, then the situation is more complicated. There may be times when $B<0$, then the potential will have one minimum point. This will enable the phase variable $x(t)$ to change sign. If then two potential wells are formed again, $x(t)$ can either return or be in another well. Therefore, we can talk about the regimes of oscillations $x(t)$ around one of the two opposite values and transitions (inversions) between them. From the point of view of dynamo theory, it will be a combination of regimes of vacillations and reversals.

Now consider the case of feedback delay: $K(0)=0$, i.e. $A=0$. The shape of the potential depending on the sign $B$ is shown in the Fig. 2. 


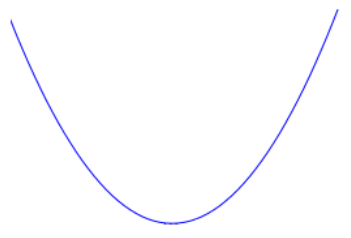

$\mathrm{B}<0$

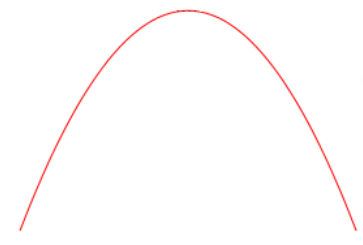

$\mathrm{B}>0$

Figure 2. The shape of potential $U(x)$ for oscillator (5) in case $K(0)=0$.

If $J(t)<0$ for all $t \geq 0$, then always $B>0$ and the phase variable $x(t)$ can be unlimited. This situation is physically unacceptable, i.e. these kernels do not make sense.

If $J(t)>0$ for all $t \geq 0$ or $J(t)$ alternating, then $B$ can also change sign. When $B>0$ there is an increase in $|x(t)|$ (Fig. 2 - right), when $B<0$ there is an decrease in $|x(t)|$ due to dissipation (Fig. 2 - left).

Therefore, the phase variable will oscillate. The form of these oscillations may depend on the rate of kernel $J(t)$ decay. This rate determines the inertia of the potential. For example, if the memory is long and $B$ stays in the negative area for a long time, $x(t)$ will be near zero for a long time. The solution will be in the nature of bursts. If the memory is short, oscillations or vacillations can be expected in solutions.

Below in the paper we will compare our reasoning with the results of numerical simulation.

\section{Numerical simulations}

Numerical simulation of the system was carried out using a specially developed implicit second-order scheme. The scheme itself is not considered in this paper. The simulation used the value $\sigma=3.37$, which is typical for Parker's dynamo. Let's look at some of the results, for examples.

\subsection{Kernel $K(t)=e^{-b t}, b>0$}

This case of the kernel corresponds to the classical Lorenz system, since

$$
z(t)=\int_{0}^{t} e^{-b(t-\tau)} x(\tau) y(\tau) d \tau
$$

equal to

$$
\frac{d z}{d t}=x y-b z, \quad z(0)=0 .
$$

The kernel $J(t)=e^{-b t}(2 \sigma-b) / 2$. In case $b>2 \sigma$ the kernel $J(t)<0$ for all $t \geq 0$, therefore $B>0$ always. As mentioned above, there should be a stable generation regime without oscillations. The results are shown in the Fig. 3.

In case $b<2 \sigma$ the kernel $J(t)>0$ for all $t \geq 0$, therefore $B$ can change sign. As mentioned above, it will be a combination of regimes of vacillations and reversals. The results are shown in the Fig. 4. It can be clearly seen that the reversals of the field is synchronized with the events $B<0$. If $B$ is positive, but close to zero, excursions can occur - short-term changes in the sign of the field.

These simulation results are in good agreement with the above qualitative analysis. 


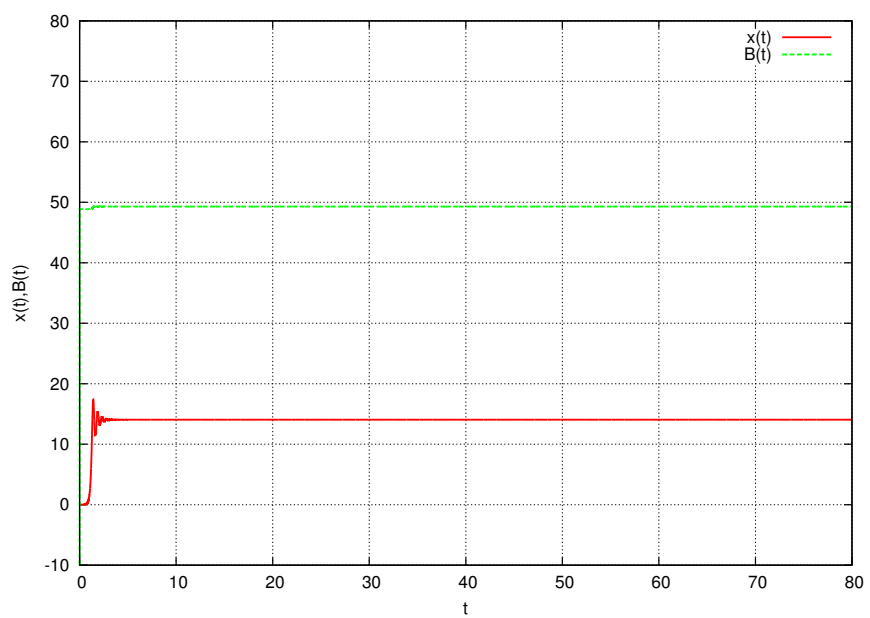

Figure 3. Simulation results. Kernel $K(t)=e^{-b t}$. Parameters: $b=6.8, D=30$.

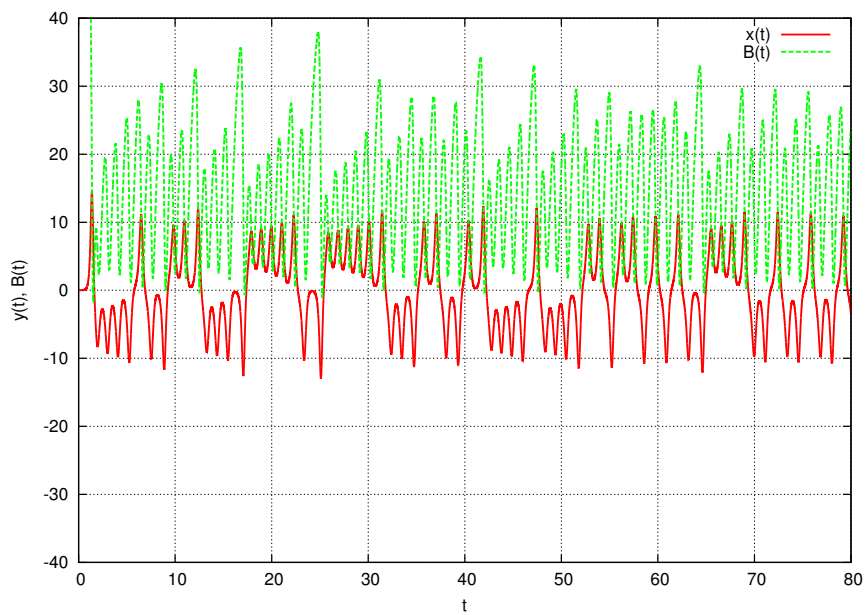

Figure 4. Simulation results. Kernel $K(t)=e^{-b t}$. Parameters: $b=1.3, D=30$.

4.2 Kernel $K(t)=t \cdot e^{-b t}, b>0$

Now let's look at one option for delaying feedback. For $K(t)=t e^{-b t}$ the kernel

$$
J(t)=e^{-b t} \frac{(2 \sigma-b) t+1}{2}
$$

will not be negative all the time, so solutions that grow indefinitely will not arise.

The simulation results are shown in Fig. 5 and 6. It is clearly seen that when $B>0$ there is an increase in filed, when $B<0$ there is an decrease. This is the behavior we discussed above.

In the Fig 5., the parameter $b=0.5$ (the kernel $J(t)$ slowly decays - long memory), therefore $B$ is delayed in the negative area and field is in the burst regime. In the Fig 6., the 
parameter $b=3.5$ (short memory). In this case, $B$ depends mainly on the values of $x(\tau)$ that are close in time $t$ and quickly responds to their changes.

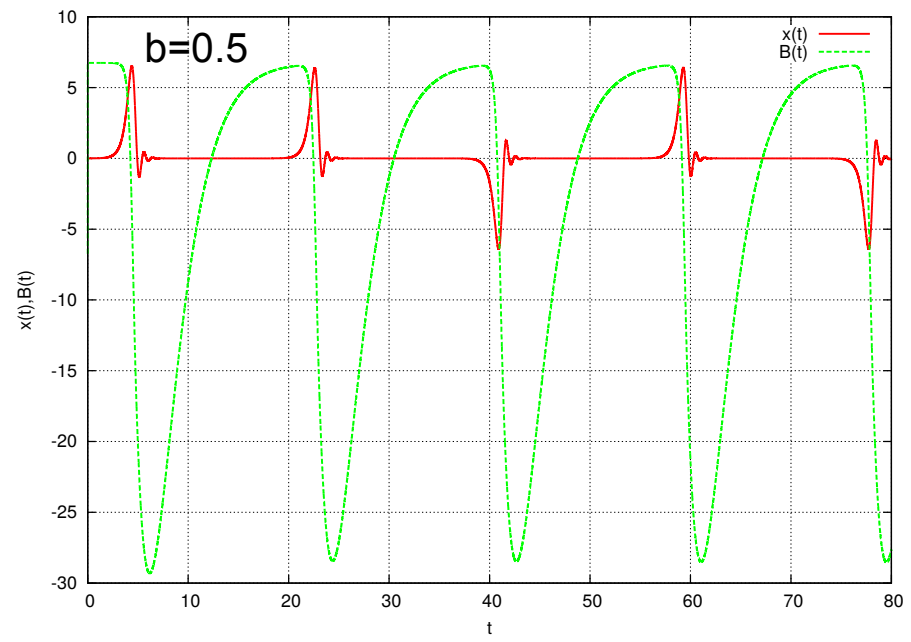

Figure 5. Simulation results. Kernel $K(t)=t e^{-b t}$. Parameters: $b=0.5, D=30$.

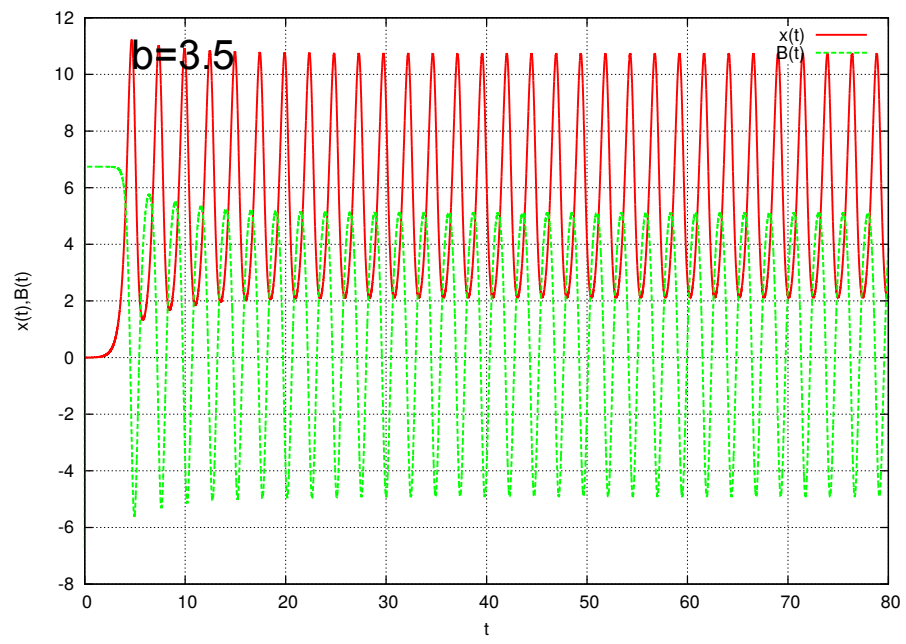

Figure 6. Simulation results. Kernel $K(t)=t e^{-b t}$. Parameters: $b=3.5, D=30$.

These simulation results for delay in feedback are in good agreement with the above qualitative analysis too.

\section{Conclusion}

The paper analyzes the previously proposed by the author model of a two-mode dynamo [6] from the point of view of the theory of oscillators with hereditary potential. It is shown that with the help of a qualitative analysis of the oscillator, it is possible to establish what 
dynamic modes will exist in the model without performing numerical simulation. It also allows for better physical meaning of the model.

\section{Acknowledgement}

The paper was carried out within the framework on the subject «Dynamics of physical processes in active zones of near space and geospheres» (AAAA-A17-117080110043-4)

\section{References}

[1] R. T. Merril, M. W. McElhinny, P. L. McFadden, The Magnetic Field of the Earth: Paleomagnetism, the Core, and the Deep Mantle (Academic Press, London, 1996).

[2] M. Stix, The Sun. An Introduction (Springer, Berlin, 2002).

[3] T. Rikitake, Proc. Cambridge Philos. Soc., 54, 89-105 (1958

[4] E. Lorenz, J. Atmos. Sci., 20, 130-141 (1963)

[5] A. A. Ruzmaikin, Comments on Astrophys, 9, 85-96 (1981)

[6] G. M. Vodinchar, E. A. Kazakov, E3S Web of Conferences, 62, 02011 (2018) https://doi.org/10.1051/e3sconf/20186202011

[7] E. N. Parker, Astrophys. J., 122, 293-314 (1955) 